APH N.S., Heavy Ion Physics 15 (2002) 000-000

HEAVY ION

PHYSICS

(C) Akadémiai Kiadó

\title{
Deformation Quantization, Superintegrability, and Nambu Mechanics
}

\author{
Cosmas K Zachos ${ }^{1}$ and Thomas L Curtright ${ }^{2}$ \\ ${ }^{1}$ High Energy Physics Division,Argonne National Laboratory, \\ Argonne, IL 60439-4815, USA \\ 2 Department of Physics, University of Miami, \\ Box 248046, Coral Gables, Florida 33124, USA \\ Received 17 October 2002
}

\begin{abstract}
Phase Space is the framework best suited for quantizing superintegrable systems - systems with more conserved quantities than degrees of freedom. In this quantization method, the symmetry algebras of the hamiltonian invariants are preserved most naturally. We illustrate the power and simplicity of the method through new applications to nonlinear $\sigma$-models, specifically for Chiral Models and de Sitter $N$-spheres, where the symmetric quantum hamiltonians amount to compact and elegant expressions, in accord with the Groenewold-van Hove theorem. Additional power and elegance is provided by the use of Nambu Brackets (linked to Dirac Brackets) involving the extra invariants of superintegrable models. The quantization of Nambu Brackets is then successfully compared to that of Moyal, validating Nambu's original proposal, while invalidating other proposals.
\end{abstract}

Keywords: deformation quantization, superintegrability, Nambu Brackets PACS: 02.30.Ik,11.30.Rd,11.30.-j

This is a pedagogical talk based on [1], with expanded context. Highly symmetric quantum systems are often integrable, and, in special cases, superintegrable and exactly solvable [2]. A superintegrable system of $N$ degrees of freedom has more than $N$ independent invariants, and a maximally superintegrable one has $2 N-1$ invariants. In the case of velocity-dependent potentials, when quantization of a classical system presents operator ordering ambiguities involving $x$ and $p$, the general consensus has long been [3] to select those orderings in the quantum hamiltonian which maximally preserve the symmetries present in the corresponding classical hamiltonian. However, even for simple systems, such as $\sigma$-models considered here, such constructions may become involved and needlessly technical.

There is a quantization procedure ideally suited to this problem of selecting the quantum hamiltonian which maximally preserves integrability. In contrast to con- 
ventional operator quantization, this problem is addressed most cogently in Moyal's phase-space quantization formulation $[4,5]$, reviewed in [6]. The reason is that the variables involved in it ("phase-space kernels" or "Weyl-Wigner inverse transforms of operators") are c-number functions, like those of the classical phase-space theory, and have the same interpretation, although they involve $\hbar$-corrections ("deformations"), in general - so $\hbar \rightarrow 0$ reduces them to the classical expressions. It is only the detailed algebraic structure of their respective brackets and composition rules which contrast with those for the variables of the classical theory. This complete formulation is based on the Wigner Function (WF), which is a quasi-probability distribution function in phase-space, and comprises the kernel function of the density matrix. Observables and transition amplitudes are phase-space integrals of kernel functions weighted by the WF, in analogy to statistical mechanics. Kernel functions, however, unlike ordinary classical functions, multiply through the $\star$-product, a noncommutative, associative, pseudodifferential operation, which encodes the entire quantum mechanical action and whose antisymmetrization (commutator) is the Moyal Bracket (MB) [4-6], the quantum analog of the Poisson Bracket (PB).

Groenewold's correspondence principle theorem [7] (to which van Hove's extension is often attached [8]) points out that, in general, there is no invertible linear map from all functions of phase space $f(x, p), g(x, p), \ldots$, to hermitean operators in Hilbert space $\mathfrak{Q}(f), \mathfrak{Q}(g), \ldots$, such that the PB structure is preserved,

$$
\mathfrak{Q}(\{f, g\})=\frac{1}{i \hbar}[\mathfrak{Q}(f), \mathfrak{Q}(g)] .
$$

Instead, the Weyl correspondence map $[9,6]$ from functions to ordered operators, $\mathfrak{W}(f) \equiv \frac{1}{(2 \pi)^{2}} \int d \tau d \sigma d x d p f(x, p) \exp (i \tau(\mathfrak{p}-p)+i \sigma(\mathfrak{x}-x))$, specifies a -product $[7,6], \quad \mathfrak{W}(f \star g)=\mathfrak{W}(f) \mathfrak{W}(g)$, and thus

$$
\mathfrak{W}(\{\{f, g\}\})=\frac{1}{i \hbar}[\mathfrak{W}(f), \mathfrak{W}(g)] .
$$

The MB is defined as $\{\{f, g\}\} \equiv(f \star g-g \star f) / i \hbar$, and, as $\hbar \rightarrow 0, \mathrm{MB} \rightarrow \mathrm{PB}$. That is, it is the $\mathrm{MB}$ instead of the $\mathrm{PB}$ which maps invertibly to the quantum commutator. This relation underlies the foundation of phase-space quantization $[7,6]$.

Conversely, given an arbitrary operator $\mathfrak{F}(\mathfrak{x}, \mathfrak{p})$ consisting of operators $\mathfrak{x}$ and $\mathfrak{p}$, one might imagine rearranging it by use of Heisenberg commutations to a canonical completely symmetrized (Weyl-ordered) form, in general with $O(\hbar)$ terms generated in the process. It might then be mapped uniquely to its Weyl-correspondent cnumber kernel function $f$ in phase space $\mathfrak{x} \mapsto x$, and $\mathfrak{p} \mapsto p, \quad \mathfrak{W}^{-1}(\mathfrak{F})=f(x, p, \hbar)$. (In practice, there is a more direct inverse transform formula $[7,6]$ which bypasses a need to rearrange to a canonical Weyl ordered form explicitly.) Clearly, operators differing from each other by different orderings of their $x$ s and ps correspond to kernel functions $f$ coinciding with each other at $O\left(\hbar^{0}\right)$, but different in $O(\hbar)$, in general. Thus, a survey of all alternate operator orderings in a problem with such ambiguities amounts, in deformation quantization, to a survey of the "quantum correction" $O(\hbar)$ pieces of the respective kernel functions, ie the inverse Weyl transforms of 
those operators, and their study is greatly systematized and expedited. Choice-ofordering problems then reduce to purely $\star$-product algebraic ones, as the resulting preferred orderings are specified through particular deformations in the c-number kernel expressions resulting from the particular solution in phase space.

In this phase-space quantization language, Hietarinta [10] has investigated the simplest integrable systems of velocity-dependent potentials. In each system, he has promoted the vanishing of the PB of the classical invariant $I$ (conserved integral) with the hamiltonian, $\{H, I\}=0$, to the vanishing of its $\mathrm{MB}$ with the hamiltonian, $\left\{\left\{H_{q m}, I_{q m}\right\}\right\}=0$. This dictates quantum corrections, addressed perturbatively in $\hbar$ : he has found $O\left(\hbar^{2}\right)$ corrections to the $I$ s and $H$, needed for quantum symmetry. The specification of the symmetric hamiltonian then is complete, since the quantum hamiltonian in terms of classical phase-space variables corresponds uniquely to the Weyl-ordered expression for these variables in operator language.

We quantize nonlinear $N$-sphere $\sigma$-models and chiral models algebraically, to argue for the general principles of power and convenience in isometry-preserving quantization in phase space: The procedure of determining the proper symmetric quantum Hamiltonian yields remarkably compact and elegant expressions. Briefly, we find [1] that the maximal symmetry generator invariants are undeformed by quantization, but the Casimir invariants of their MB algebras are deformed, in accord with the Groenewold-van Hove theorem. Hence, the hamiltonians are also deformed,

$$
H_{q m}-H=\frac{\hbar^{2}}{8}(\operatorname{det} g-1-N(N-1)),
$$

for $S^{N}$, where det $g$ is the determinant of the hypersphere metric in the orthogonal projection, and the spectra are seen to be proportional to $l(l+N-1)$ for integer $l$. For $G \times G$ chiral models, we find, for the corresponding group manifold quantities,

$$
H_{q m}-H=\frac{\hbar^{2}}{8}\left(\Gamma_{a c}^{b} g^{c d} \Gamma_{b d}^{a}-f_{i j k} f_{i j k}\right) .
$$

Quantization of maximally superintegrable systems in phase space has an unexpected application: it facilitates explicit testing of Nambu Bracket (NB) [11] quantization proposals, through direct comparison to the conventional quantum answers thus found.

The classical evolution of all functions in phase space for such systems is alternatively specified through NBs $[1,12]$, because the phase-space velocity is always perpendicular to the $2 N$-dimensional phase-space gradients of $2 N-1$ independent integrals of the motion, $L_{i}$. Thus, for an arbitrary phase-space function $k$ with no explicit time dependence, the classical evolution is fully specified by a phase-space jacobian which amounts to the Nambu Bracket:

$$
\begin{aligned}
\frac{d k}{d t} & =V \frac{\partial\left(k, L_{1}, \ldots, \ldots, L_{2 N-1}\right)}{\partial\left(q_{1}, p_{1}, q_{2}, p_{2}, \ldots, q_{N}, p_{N}\right)} \\
& \equiv V\left\{k, L_{1}, \ldots, L_{2 N-1}\right\}
\end{aligned}
$$


where the proportionality constant $V$ can be shown to be a time independent function of the invariants. E.g., on $S^{2}$,

$$
\frac{d k}{d t}=\frac{\partial\left(k, L_{x}, L_{y}, L_{z}\right)}{\partial\left(x, p_{x}, y, p_{y}\right)}
$$

where the $L_{i}$ s are invariants corresponding to translations along two orthogonal meridians and circles of latitude, respectively.

However, consistent quantization of NBs has been considered problematic ever since their inception. Nevertheless, Nambu's original quantization prescription [11] can, indeed, succeed, despite widespread expectations to the contrary. Comparison to the standard Moyal deformation quantization vindicates [1] Nambu's early quantization prescription (and invalidates other prescriptions), for systems such as $S^{N}$. E.g., for the above $S^{2}$, the MB result is actually equivalent to Nambu's quantum bracket [1],

$$
\frac{d k}{d t}=\frac{-1}{2 \hbar^{2}}\left[k, L_{x}, L_{y}, L_{z}\right]_{\star}=\left\{\left\{k, H_{q m}\right\}\right\},
$$

where $[A, B]_{\star} \equiv i \hbar\{\{A, B\}\}$ and

$$
\begin{aligned}
{[A, B, C, D]_{\star} \equiv } & A \star[B, C, D]_{\star}-B \star[C, D, A]_{\star}+C \star[D, A, B]_{\star}-D \star[A, B, C]_{\star} \\
= & {[A, B]_{\star} \star[C, D]_{\star}+[A, C]_{\star} \star[D, B]_{\star}+[A, D]_{\star} \star[B, C]_{\star} } \\
& +[C, D]_{\star} \star[A, B]_{\star}+[D, B]_{\star} \star[A, C]_{\star}+[B, C]_{\star} \star[A, D]_{\star}
\end{aligned}
$$

It turns out that certain conditions often posited and not easy to satisfy in general in NB quantization schemes are either satisfied automatically for these superintegrable systems, or the quantization goes through consistently despite failing such unnecessary conditions. We thus stress the utility of phase space quantization as a comparison testing tool for NB quantization proposals. More elaborate isometries of general manifolds in such models are expected to yield to analysis similar to what has been illustrated for prototypes.

\section{Acknowledgements}

We gratefully acknowledge helpful questions from R Sasaki, D Fairlie, Y Nutku, V Fleurov, J-P Dahl, and J Klauder. This work was supported in part by the US Department of Energy, Division of High Energy Physics, Contract W-31-109-ENG38, and the NSF Award 0073390.

\section{References}

1. T Curtright and C Zachos, [hep-th/0205063] New J Phys, in press.

2. P Tempesta, A Turbiner, and P Winternitz, J Math Phys 42 (2001) 4248-4257 [hep-th/0011209]; M Rodriguez and P Winternitz, J Math Phys 43 (2002) 1309-1322 [math-ph/0110018]; E Kalnins, J Kress, and P Winternitz, $J$ Math Phys 43 (2002) 970-983 [math-ph/0108015]. 
3. G Velo and J Wess, Nuov Cim A1 (1971) 177; H Lin, W Lin, and R Sugano, Nucl Phys B16 (1970) 431; M Lakshmanan and K Eswaran, J Phys A8 (1975) 1658; also see A Leznov, Nucl Phys B640 (2002) 469-480 [hep-th/0203225]; P Higgs, J Phys A12 (1979) 309; H Leemon, J Phys A12 (1979) 489.

4. J Moyal, Proc Camb Phil Soc 45 (1949) 99-124.

5. T Curtright, D Fairlie, and C Zachos, Phys Rev D58 (1998) 025002.

6. C Zachos, Int J Mod Phys A17 (2002) 297-316 [hep-th/0110114]; J P Dahl, Adv Quantum Chem 39 1-18 (2001).

7. H Groenewold, Physica 12 (1946) 405-460; also see B Leaf, J Math Phys 9 (1968) 65.

8. L van Hove, Proc R Acad Sci Belgium 26 1-102 (1951).

9. H Weyl, Z Phys 46 (1927) 1; also reviewed in H Weyl, The Theory of Groups and Quantum Mechanics, Dover, New York, 1931.

10. J Hietarinta, J Math Phys 25 (1984) 1833-1840.

11. Y Nambu, Phys Rev D7 (1973) 2405-2412.

12. J Hietarinta, J Phys A30 (1997) L27-L33; N Mukunda and E Sudarshan, Phys Rev D13 (1976) 2846-2850; R Chatterjee, Lett Math Phys 36 (1996) 117-126; Y Nutku, "Quantization with Nambu-Poisson brackets", preprint; C Gonera and Y Nutku, Phys Lett A285 (2001) 301-306. 